\title{
El profesorado y el cambio educativo. Consideraciones teóricas y metodológicas
}

\author{
Xavier Bonal \\ Universicat Autónoma de Barcelona. Departament de Sociologia \\ 08193 Bellacerra (Barcelona). Spain
}

\section{Resumen}

Las teorlas dominantes en sociología de la educación no consiguen resolver interrogantes fundamentales para explicar el cambio educativo, tanto en relación con el origen del cambio como por lo que respecta al papel de los agentes educativo en dicho proceso. Las aportaciones de la teorla de las resistencias permiten conceptualizar teóricamente el papel del profesorado en los procesos de cambio, pero presenta limitaciones metodológicas importantes, las cuales pueden ser relativamente superadas por la investigación-acción crítica. A partir de las reflexiones desarrolladas en este artículo es posible construir las bases téricas para el desartollo de una metodología de inducción del cambio, en la que sea posible distinguir entre actitudes y prácticas reproductoras y contrahegemónicas. (Autor)

Palabras clave: actitudes del profesorado, cambio educativo, cambio cultural, búsquedaacción.

\section{Abstract. Teacher's role in educational change processes. Theoretical and methodological considerations}

Main contemporary theories in sociology of education cannot explain most causes and features of educational change. This concerns to the origins of educational change as well as to the agents' role in the process. Contributions of theories of resistance count for a theoretical explanation of teachers' role in educational change processess, but they lack a rigourous methodological developmenr about how this is to be done. Critical action-research may overcome these limitations, basically through a new definition of teachers-facilitator relationship. Reflections presented in this article allow us to build the theoretical bases to develop a methodolgy for educational change in schools, in which it is possible to distinguish reproductive and counterhegemonic practices. (Author)

Key words: teachers attitudes, educational change, cultural change, action-research. 


\section{Sumario}

Introducción Valoración de la investigación-acción

La teoria de las resistencias y el cambio educativo critica e implicaciones para el cambio educarivo

La investigación-acción como mérodo aplicado a la educación

Bases para una metodología de intervención para el cambio educativo

Las limitaciones de la investigación-acción dominante para el cambio educarivo

La investigación-acción crítica

Conclusiones

Bibliografia

\section{Introducción}

El estudio sobre las expectativas y actitudes del profesorado en el contexto educativo ha sido un aspecto bastante olvidado por la sociologia de la educación. En general, se ha considerado un ámbito de estudio de la psicología dentro de las ciencias de la educación. Ello ha conllevado que este análisis se individualice, y por lo tanto se omita su dimensión sociológica; es decir, el análisis de los factores sociales y contextuales que estructuran unas prácticas sociales determinadas de un colectivo profesional, y el desvelamiento de los universos simbólicos en los que se enmarcan tales prácticas.

Por otra parte, en nuestro país, cuando la sociología de la educación se ha ocupado del estudio del profesorado, lo ha hecho analizando su situación como colectivo profesional en la estructura social. El análisis de sus posiciones ideológicas (su "progresismo" o "conservadurismo") se ha relacionado con variables independientes como el origen social, el sexo, la edad o el tipo de centro escolar en el que trabaja, etc. (Masjoan, 1974; Varela y Ortega, 1984). Estudios más recientes se han ocupado del análisis de las causas de la baja sindicación del colectivo docente, en base a sus actitudes frente a la ocupación que desempeñan, las cuales se mueven de forma contradictoria entre el profesionalismo y la proletarización y ante las que no existe una definición institucional clara a nivel sindical (Guerrero, 1990).

Es precisamente la posición contradictoria del profesorado entre el profesionalismo y la proletarización la tesis que centra los trabajos sobre los ensenantes en la sociología de la educación anglosajona de los ochenta (Apple, 1986; Ball, 1988; Grace, 1985; Lawn, 1987; Ozga y Lawn, 1988). La tendencia a la proletarización se expresa a partir de la creciente separación entre concepción y ejecución del trabajo docente (mayor especialización de las agencias de control), así como a partir de una mayor intensificación del mismo. El profesorado, como orros colectivos profesionales, sufre entonces un proceso de descualificación en la medida en que pierde autonomía sobre su trabajo. Este proceso, señala Apple (1986: 45), queda oculto por la mayor especialización de tareas y de conocimientos en los centros escolares, lo que conilleva una percepción subjetiva de mayor profesionalismo.

Por orra parte, estos estudios también nos indican como no solamente deben considerarse las posiciones de clase en el análisis del profesorado. El 
hecho de tratarse de un colectivo mayoritariamente femenino tiene consecuencias básicas para el análisis del mismo. El proceso de proletarización debe entenderse en un contexto institucional en el que la fuerza de trabajo está constituida principalmente por mujeres y los puestos de dirección están ocupados por hombres. Ello explica, por ejemplo, como el profesionalismo constituye una salida para las mujeres enseñantes, que se ven sometidas a una doble presión: la que es ejercida por el Estado sobre el control de su trabajo y la que se deriva de la dominación masculina en la esfera doméstica.

La validez de este análisis no es discutible para explicar los procesos y formas que toman tanto el proceso de trabajo como las luchas políticas y resistencias del colectivo docente (aunque sí sería discutible la validez de la tesis de la proletarización en su aplicación a la situación del profesorado en Cataluña, teniendo en cuenta su evolución histórica), pero sí lo es cuando se toma como punto de partida para tratar de explicar las posibilidades de cambio educativo que se encierran en la posición contradictoria del profesorado. No hay duda de que la existencia de contradicciones sienta las bases para el cambio político. En el caso del profesorado, las contradicciones de clase y de género pueden ser factores, y de hecho en determinadas ocasiones lo son, capaces de movilizar al profesorado hacia determinadas acciones de lucha. Otra cosa, sin embargo, es considerar los efectos de las posiciones estructurales contradictorias sobre la transmisión cultural. Es decir, conviene efectuar la distinción entre la disposición a la lucha y al cambio debida a la experimentación personal de la discriminación por razón de clase o género, y la disposición a modificar un discurso y unas prácticas que pueden ser clasistas o sexistas.

No quiero decir con esto que se trate de cuestiones completamente independientes, pero sí poner de relieve la insuficiencia de la explicación de que el cambio pueda emerger en el momento en que las contradicciones que existen sobre la educación no resistan la tensión a la que están sometidas. Porque, si el cambio dependiera exclusivamente de esto, ¿en qué momento podría y debería producirse, es decir, en qué momento la tensión sería insostenible?, ¿qué direcciones tomaría?, ¿qué agentes generarían el "lengutaje de la posibilidad» (Apple, 1986; Giroux, 1990)?

Nada hace pensar que no existan mecanismos a disposición del Estado que permitan mitigar las situaciones de conflicto, sin que se altere por ello la reproducción de relaciones de clase y de género. Los incrementos salariales al colectivo docente, las atenciones a las demandas sobre la jornada de trabajo intensiva, o incluso la reforma educativa son buenas pruebas de ello.

La teorfa educativa neomarxista sitúa acertadamente las contradicciones en el punto de partida para el cambio posible (Bowles y Gintis, 1976; Carnoy y Levin, 1985). La reproduccion, nos recuerdan, no es solamente la de las necesidades del capital y del Estado. Es también la reproducción de las contradicciones y de los conflictos. Otros autores insisten además en que éstas existen de forma relativamente autónoma en las esferas económica, política y cultural (Apple, 1982; 1987). 
Pero es el paso siguiente el que queda por resolver; es decir, situar en algún espacio el origen del cambio así como las estrategias metodológicas para llevarlo a cabo. Las limitaciones de los teóricos radicales se evidencian en el momento de "bajar a la arena" e intentar describir en qué medida la institucion escolar es una institución cultural reproductora de relaciones de clase y de género.

En definitiva, mientras es acertado considerar que las contradicciones y la conciencia de opresión están en la base de la formación de los movimientos sociales, lo es menos pensar que cualquier situación de experimentación de las contradicciones culturales o políticas genera una actitud de lucha y una disposición al cambio político, y menos aún que la aparición de esa actitud se produzca a través de la transmisión de un discurso unidireccional. Ello equivaldría a confundir la posición objetiva y subjetiva de los individuos en la estructura social y a pensar que la "conciencia de conflicto" (Giddens, 1979) puede alcanzarse a través de un proceso simple de persuasión política.

Por lo tanto, el análisis de las posibilidades y límites del cambio educativo, entendido como cambio en las ideologias y en las prácticas de los agentes de transmisión cultural, debe situarse al nivel del trabajo cotidiano del profesorado, en el nivel de las formas posibles que puede tomar la deconstrucción y reconstrucción de la cultura escolar. Lo que no está resuelto en la teoría educativa crítica es la respuesta a los interrogantes siguientes: ¿Bajo qué condiciones es posible el cambio educativo? ¿Cuándo este cambio constituye una simple alteración y cuándo es real? ¿Son las contradicciones identificables en el nivel cultural suficientes para que se produzcan prácticas transformadoras? ¿Hasta qué punto para que el cambio sea posible tiene que existir un paralelismo entre el discurso que se transmite y la propia vivencia de una situación estructural contradictoria? ¿Como y cuándo pasa el propio profesorado de transmitir el discurso dominante a producir el discurso que transmite? ¿Como puede el profesorado acceder y considerat los elementos de las culturas dominadas para introducirlos en el currículo?

Desde la teoría de las resistencias se ha procurado dar respuesta a estas cuestiones.

\section{La teoría de las resistencias y el cambio educativo}

Aunque sin una única formulación, la teoría de las resistencias (Giroux, 1981; 1983; 1990; Anyon, 1981; 1983; Willis, 1977; 1986) constituye una alternativa teórica a las formulaciones más estructuralistas de la teoría de la reproducción. El planteamiento se centra en la consideración de que la escuela y los propios agentes educativos no son solamente agentes portadores de un discurso y unas prácticas que reproducen el orden social, sino que son un espacio y unos grupos capaces de producir nuevos conocimientos $y$, por consiguiente, prácticas alternativas.

Se trata, por lo tanto, de dar cabida a la posibilidad de cambio educativo, cambio que puede no tener su único origen en las contradicciones que 
viven los agentes educativos entre su posición en la estructura social en cuanto a género y clase social y la realización de unas prácticas que se estructuran a partir de un discurso que ignora la diversidad social y cultural. Sin duda, es Giroux el autor que más se ha preocupado por desarrollar un nuevo discurso teórico alternativo que siente las bases para el cambio posible. Giroux señala como profesores y alumnos pueden constituir grupos capaces de comportarse como intelectuales que pueden desarrollar prácticas y conocimientos contrahegemónicos. Puede existir, por lo tanto, alguna forma de potenciación pedagógica capaz de hacer desarrollar prácticas sociales alternativas.

El papel del profesorado y de la investigación educativa, según Giroux, debe definirse en términos del papel que deben desarrollar como intelectuales transformativos y a ellos les corresponde reconsiderar y transformar las condiciones materiales en las que se desarrolla su trabajo.

En esta línea, Girotix (1990) también propone las bases para el desarrollo de un nuevo currículo que incorpore las vidas y los conocimientos de los grupos dominados. La diferencia cultural se convierte entonces en la clave a partir de la cual el profesorado puede desarrollar un cambio, pero para ello debe luchar contra una institución con voluntad de homogeneización, de normalización cultural. Debe trabajar para entender las diferentes lógicas culturales y conocer como estas logicas acrúan dentro de relaciones asimétricas de poder. Ello implica sobrepasar el discurso de la integración y de la pluralidad, discurso que Giroux califica de neoliberalismo a partir del cual es imposible alcanzar la igualdad educativa. La igtaldad educativa se consigue solamente a través de la incorpotación de las diferencias culturales a un nivel de visibilidad. Comporta traspasar el nivel del pluralismo y saber comprender la forma en que se expresan las formas culturales de los grupos dominados en las relaciones asimétricas de poder.

La posible vía de cambio está en una lucha democrática, en una lucha encaminada hacia la constitución de una escuela como esfera pública democrática de producción cultural. En sus últimas publicaciones, Giroux (1992) extiende su idea de pedagogía radical a partir del desarrollo de una pedagogía de los limites, una pedagogía que debe desarrollarse como política cultural de la diferencia y que haga posible la penetración de las culturas dominadas en los "supuestos límites homogéneos y sin problemas de las formas y prácticas culturales dominantes" (Giroux, 1992: 153).

La teoría de las resistencias nos proporciona dos elementos esenciales para el análisis de los procesos de cambio en la escuela. En primer lugar, desarrolla la idea de posibilidad de que el profesorado y el alumnado desarrollen prácticas sociales alternativas. El origen del cambio, en este sentido, no se sitúa necesariamente en las contradicciones que los individuos viven entre su posición en la estructura social (de clase, raza o género) y su práctica en una institución de reproducción social y cultural. "Es posible que la posición de clase, raza, género o etnia suponga algún tipo de influencia, pero no predetermina irrevocablemente la ideología que uno adopta, la forma en que se interpreta un 
texto determinado o como se responde a determinadas formas de opresión" (Giroux, 1992: 146).

En segundo lugar, la teoría de las resistencias da un paso importante al considerar que la igualdad educativa se alcanza solamente a partir de la incorporación de las diferencias culturales en el currículo. La igualdad, por lo tanto, no es dar a todo el mundo lo mismo. La igualdad educativa pasa por introducir en el currículo lo que la forma cultural dominante oculta. Ello conlleva necesariamente la adopción de una postura crítica respecto a las prácticas educativas cotidianas, y reestructurarlas hacia un objetivo de transformación de las condiciones materiales de trabajo. En definitiva, la igualdad educativa basa$\mathrm{d} a$ en las diferencias culturales solamente se alcanza a través de la politización del trabajo pedagógico.

Estas dos consideraciones, como veremos, son fundamentales para la construcción de una metodología para el estudio sociológico del cambio educativo. Es decir, para la fijación de las condiciones bajo las cuales el cambio educativo puede tener lugar.

La gran limitación de la teoría de las resistencias es precisamente no haber desarrollado dicha metodología. No solamente para posibilitar la interpretación de qué prácticas o manifestaciones de resistencia se oponen a las prácticas educativas dominantes, sino también para definir una forma de colaboración entre agentes externos e internos a la institución escolar, a partir de la cual se desarrolle un proceso de cambio. La investigación-acción nos da alguna respuesta a estas limitaciones metodológicas.

\section{La investigación-acción como método aplicado a la educación}

La investigación-acción, como metodología aplicada al terreno educativo, aparece en el mundo anglosajón a mediados de los setenta. Surge como alternativa a las metodologías aplicadas hasta entonces al desarrollo curricular y al cambio otganizativo-institucional. Modelos como el "Centro-periferia» o el modelo "Investigación-Desarrollo-Difusión" (ID y D) dominantes en el mundo anglosajón, fracasan como sistemas eficaces de mejorar la calidad de la enseñanza?

A mediados de los setenta, y básicamente a partir de las ideas de Lawrence Stenhouse (1981) sobre el papel investigador del profesorado, empiezan a impulsarse proyectos de investigación-acción, de entre los cuales conviene destacar los del propio Stenhouse (Humanities Curriculum Project) y los de John Elliott (1990). La investigación en la acción supone un cambio importante, tanto en lo que se refiere a la conceptualización del trabajo profesional del profesorado como respecto a la relación entre teoría o conocimiento educativo y práctica o acción educativa.

Por una parte, la concepción del tol del profesor como profesional que dispone de un conjunto de conocimientos teóricos con el objetivo de transmitir

1. Para una crítica de estos modelos, véase Popkewizz (1988). 
unos determinados saberes, desligado del propio proceso de descubrimiento del conocimiento, conlleva una relación determinada entre teoría y práctica, una relación en la que la teoría marca los principios de la acción, mientras se mantiene en todo momento por encima de la misma. Según este enfoque, lo que se da por supuesto es una identificación entre las teorías que subyacen a unas determinadas prácticas educativas y la conciencia que los agentes educativos tienen de las teorías que orientan sus prácticas. Es decir, se produce una identificación entre los valores subjetivos que orientan la acción y los principios subyacentes a la práctica. Desde este punto de vista, cuanto mayor es el conocimiento tebrico a disposición del agente educativo, más eficaz es el proceso de transmisión cultural.

Por otra parte, desde este enfoque se separa completamente el rol de investigador del rol de "práctico". Los agentes que lo llevan a cabo son profesionales distintos: los especialistas del conocimiento educativo investigan sobre la educación y aportan sus nuevos conocimientos en forma de innovaciones técnicas para ser aplicadas por los profesionales encargados de la práctica.

La investigación-acción incorpora una nueva concepción de la profesión educativa y una redefinición de la relación entre teoría y práctica, lo que aleja a este enfoque de las posiciones técnico-positivistas anteriores. La centralidad del trabajo de investigación sobre la propia práctica hace posible que a través de la exploración de las diferentes situaciones educativas y de la contrastación de hipótesis, pueda emerger el sesgo entre la teoría y los efectos de la práctica. Los agentes educativos descubren de este modo los límites y los uefectos no deseados" de su práctica; a partir de lo que han investigado planifican los cambios necesarios y los experimentan. La evaluación de la experimentación permite comprobar los nuevos efectos y reformular la situación problemática inicial.

A través de la investigación el profesorado desarrolla entonces su capacidad reflexiva crítica, y a partir de ésta su capacidad de intervención sobre su trabajo cotidiano. La incorporación de la lógica del proceso de investigación cientifica a la práctica docente cotidiana acaba por confundir en una misma práctica la acción y la evaluación sobre la misma, gracias a un ejercicio constante de reflexión. De este modo, la modificación de unas determinadas prácticas sociales es percibida como posible por parte del profesorado, y el desarrollo profesional se favorece a rravés de este incremento de autonomía sobre lo que debe enseñarse y cómo debe enseñarse.

Cabe preguntarse entonces hasta qué punto el profesorado, a través de su participación en procesos de investigación-acción puede desarrollar el papel clave de agente de cambio educativo. Si efectivamente puede modificar sus formas de pensar y sus formas de acción. De ser así, ello completaría las deficiencias empiricas y metodológicas de las distintas formulaciones de la teorfa de las resistencias educativas. Sería pertinente, en consecuencia, considerar si los posibles cambios pueden emerger de posibles situaciones de conflicto identificables en el nivel cultural. $Y$, por lo tanto, discernir aspectos sobre la modificación de prácticas. 


\section{Las limitaciones de la investigación-acción dominante para el cambio educativo}

La cuestión que se plantea es entonces si la investigación-acción, como método asociado a un modelo que se basa en el proceso más que en el producto (objetivos), responde a los interrogantes que planteábamos anteriormente. Es evidente que al poner énfasis en el desarrollo de la capacidad reflexiva del profesorado, en el análisis en profundidad sobre la propia práctica, la investigaciónacción constituye una metodologia que a priori puede completar las deficiencias empíricas de la teoría de las resistencias. En este sentido, la aplicación de este tipo de metodología en los centros educativos debería permitir desenmascarar los contenidos discriminatorios de tipo sexista, racista o clasista.

Sin embargo, la corriente de investigación-acción dominante tiene algunas limitaciones importantes. Estas limitaciones se deben básicamente a un planteamiento excesivamente técnico-metodológico, en el que existen algunas limitaciones. Estas limitaciones son de tres tipos: político, sociológico y cultural. De ellas se deducen implicaciones conceptuales y metodologicas importantes. Por una parte, poniendo de relieve estas deficiencias veremos como podemos definir el concepto de cambio educativo y distinguirlo de variación o reforma. En segundo lugar, una metodología de investigación-acción educativa debe explicitar si persigue el objetivo de cambio, $y$, como consecuencia, fijar las bases teóricas que pongan de relieve las condiciones bajo las cuales ese cambio puede tener lugar.

Podemos distinguir tres tipos de limitaciones en la LA:

a) Limitaciones políticas: $\mathrm{Al}$ centrarse básicamente en el proceso, como alternativa a los modelos de desarrollo curricular dominantes (modelos por objetivos), la investigación-acción omite las orientaciones políticas en el planteamiento de cambio educativo. Algunos autores (Elliott, 1990) subrayan la importancia de las "implicaciones políticas" que determinadas decisiones asociadas a los procesos de investigación-acción pueden tener. Al señajarío de este modo, se está poniendo en segundo plano la naturaleza política de las decisiones educativas. Lo político son posibles secuelas derivadas de decisiones estrictamente técnicas. Whitty (1985) ha criticado las limitaciones políticas de muchos enfoques de cambio, en los que las relaciones de poder y la ideología permanecen fuera del ámbito de análisis. Hargreaves (1982) por su parte, ha destacado como, en Gran Bretaña, las diferentes alternativas a la innovación educativa administrativamente centralizada (innovaciones centradas en la escuela), están sesgadas ideológicamente. Aunque Hargreaves no incluya directamente en su crítica las diferentes formas de investigación-acción dominantes, el tipo de limitaciones políticas que sufren este conjunto de aproximaciones al cambio curricular y educativo son, hasta cierto punto, similares: dan por descontado la universalidad de la idea de participación del profesorado en los proyectos de cambio, omiten los conflictos y luchas internos de la insti- 
tución escolar y se alejan del dirigismo del cambio "centro-periferia" sin apartarse al mismo tiempo de los conceptos y métodos utilizados por éste (Hargreaves, 1982: 258).

Los enfoques de cambio educativo basados en la propia escuela como centro de decisiones, entre los que cabría incorporar ciertas formas de investigación-acción, ocultan bajo una apariencia de sistemas democráticos y liberales el contenido ideológico y político que tienen per se las decisiones educativas. El énfasis que algunos planteamientos de investigación-acción educativa ponen en el propio proceso metodológico que debe orientar la actividad educativa, tiene entonces consecuencias negativas, ya que al no definir ningún tipo de objetivo explícito en términos políticos se encierran en un reformismo liberal, aparentemente progresista, pero en definitiva más reproductor que transformador.

b) Limitaciones sociológicas: Esta limitación cabe adjudicarla más a la sociologia que a la propia investigación-acción. La tradición de la sociología ha consistido en mantenerse siempre fuera de los campos de acción para el cambio. La participación o la colaboración del sociólogo en los procesos de modificación o transformación de la realidad ha sido considerada generalmente como un proceso de pérdida de rigor cientifico. La postura dominante es que la sociología debe analizar la realidad, pero no intervenir sobre ella.

Estas consideraciones permitirian reabrir el inagotable debate sobre el papel de sociología frente a la realidad social, los procesos de distanciamiento epistemológico y de ruptura con la evidencia social. Aunque este no es el momento de discurrit sobre estas cuestiones, sí me parece oportuno señalar dos tipos de consideraciones básicas que tienen consecuencias sobre las decisiones metodológicas posteriores.

En primer lugar, la polarización de posiciones es absurda. No se trata de intervenir o no intervenir, sino de como intervenir sobre la realidad, cuestión que consideraremos más adelante. La intervención sobre la realidad no tiene porque ser sinónimo de imposición ideológica. A partir de una definición clara de objetivos políticos, existe un abanico de posibilidades de inducción de los procesos de cambio, incorporando cada una de ellas diferentes niveles de autonomía de actuación de los propios actores sociales y diferentes tipos de relación entre los agentes internos de la institución y los agentes externos.

En segundo lugar, conviene distinguir entre los procesos de intervención sobre la realidad y la ruptura epistemologica que la sociología debe realizar ante el objeto de estudio. En este sentido, puede apuntarse que del mismo modo que la no intervención sobre la realidad social no garantiza que el investigador efectúe adecuadamente su "vigilancia epistemológica» (Bourdieu, 1976), puede existir un proceso de inducción controlada sobre las acciones de los actores sociales que permita al investigador situarse en una posición doble que le permita a la vez inducir situaciones de cambio y 
poder después distanciarse durante la fase de análisis. Touraine (1978), a través del desarrollo del método de intervención sociológica, ha demostrado sobradamente como esto es sociológicamente posible.

En cualquier caso, el hecho de que los sociólogos de la educación no se hayan interesado por participar en procesos de investigación-acción ha tenido consecuencias negativas, tanto sobre el desarrollo de la capacidad sociologica crítica del profesorado como sobre las posibilidades de enriquecimiento del propio análisis sociológico de la educación. Lo primero se pone de relieve al constatar como el discurso que la propia escuela realiza sobre la definición de la problemática escolar tiene una orientación claramente psicopedagógica, no sociológica (Gimeno Sacristán, 1983). De este modo, los problemas se individualizan constantemente: cada alumno o alumna requiere un tratamiento específico porque su problema es personal. La variable grupo social se omite de entre las variables explicativas de los problemas del alumnado, sea ésta la clase social, el sexo o la raza. Asimismo, la inexistencia de capacidad de análisis sociológico en el profesorado significa una falta de visión global de la proyección de valores y normas sociales externos a la institución escolar sobre las relaciones sociales que tienen lugar dentro de la propia institución.

Por otra parte, a los trabajos de investigación-acción les falta, en general, el complemento de la perspectiva sociológica. De esto, sin duda, son más culpables los propios sociólogos que los profesionales que han llevado a cabo los proyectos de investigación-acción. En este sentido, la sociología de la educación ha perdido una oportunidad inmejorable para analizar empiricamente los límites y las posibilidades del cambio educativo, tanto a nivel de ideologías y actitudes como a nivel de prácticas. Es decir, la posibilidad de contrastar empíricamente si las resistencias a las formas de dominación pueden traducirse en actirudes y prácricas contrahegemónicas, y distinguir posteriormente entre prácticas reproductoras de situaciones y relaciones simbólicas y prácticas productoras de nuevas situaciones y nuevas relaciones simbólicas. Por último, la posibilidad de inducir procesos de cambio permite poder evaluar tanto su nivel de legitimidad como la orientación y dirección que toma éste (diferencia entre cambio lineal y cambio dialéctico) a partir de sus características.

c) Limitaciones culturales: Lo que denomino limitaciones de tipo cultural son una consecuencia de las limitaciones sociológicas. La investigaciónacción dominante no sólo omite la variable grupo como explicación posible de determinadas situaciones educativas, sino que no considera la diversidad cultural que incorporan los distintos grupos sociales, ni como unas determinadas condiciones de existencia pueden explicar la producción de unas prácticas culturales concretas, diferenciadas de las prácticas culturales dominantes.

Como señala Hargreaves (1988) para el caso inglés y galés (aunque perfectamente aplicable al caso español), la competencia profesional se mide 
a partir de la capacidad de aplicación técnica de nuevas metodologías, de nuevos conocimientos, y por la capacidad personal de control y gestión sobre las diferentes situaciones educativas. No se mide, en cambio, por lo que podría denominarse competencia cultural, que permitiría al profesorado relativizar la apariencia neutral del sistema, entender éste corno un producto de su propia historia y saber sobrepasarlo críticamente.

Tener competencia cultural equivaldría a una acentuación de la percepción de las restricciones materiales que limitan sus prácticas y, al mismo tiempo, una mayor percepción de las características de formas culturales dominadas que no están incorporadas en el currictulum escolar. Ello debería permitir la captación de valores y prácticas sociales de clase, raza o género, que pasan desapercibidas ante el profesorado, o cuyas manifestaciones más evidentes son directamente reprimidas.

La mayor parte de los proyectos de investigación-acción educativa, al no explicitar (y dar por supuestos) ni los objetivos de igualdad social, ni una concreción del significado de igualdad y justicia social (de cómo ésta igualdad debe ser alcanzada), no escapan a la ideología educativa dominante, que confunde la consecución del principio de igualdad con la homogeneidad de la oferta educativa. De este modo, en las escuelas persiste un modelo ideológico que imposibilita la penetración de otros elementos culturales en las aulas y, por lo tanto, conduce a la uceguera cultural" del profesorado. Como veremos, la inducción externa en el proceso de investigación-acción es en este sentido clave para la potenciación de la capacidad de percepción cultural del profesorado.

En definitiva, una forma válida de escapar a los planteamientos apolíticos y ateóricos de algunas formas de investigación-acción y de otras estrategias de innovación y cambio educativo consiste en construir una metodología que supere los tres conjuntos de limitaciones señalados. Solamente a partir de esta superación se hace posible el establecimiento de una estrategia de intervención educativa que se plantee el cambio educativo en términos de transformación, una estrategia que potencie la capacidad de autorreflexión crítica para el cambio de las situaciones, hechos y comprensiones educativas de los propios actores. Como veremos a continuación, la investigación-acción crítica nos proporcionará el marco adecuado para esta superación. Por otra parte, destacaremos también algunas de las contradicciones entre los principios teóricos de la investigación crítica y su traducción en la práctica. Estas reflexiones fijarán las bases para la construcción de una metodología de intervención educativa más coherente con los principios teóricos.

\section{La investigación-acción crítica}

Alejándose de planteamientos positivistas e interpretativos, la investigaciónacción es, según Carr (1990), algo más que una metodología. La investigación-acción constituye en sí misma una ciencia educativa crítica, en la que la 
teoría no es separable ni diferenciable de la práctica, ya que implica un nivel de compromiso del investigador en los propios objetivos educativos. Lo que la diferencia básicamente de las ciencias anteriores es que se trata de una ciencia para la educación y no sobre la educación. Una ciencia educativa crítica tiene entonces el objetivo de transformar la educación, se dirige al cambio, y no simplemente a las explicaciones "objetivas" de los fenómenos educativos, objetivo del paradigma positivista, o a las comprensiones de los actores, finalidad del paradigma interpretativo (Carr y Kemmis, 1986: 156).

La investigación-acción, como ciencia educativa crítica, enfatiza el proceso de concienciación como proceso clave para el cambio, a través del cual los sujetos conocen a fondo su propia realidad y adquieren la capacidad necesaria para transformarla. Esta posibilidad ocurre a partir de la autorreflexión crítica que los actores realizan sobre sus propias prácticas, y la transformación de tales prácticas a través de un proceso de constante participación y colaboración. Tal y como señalan Carr y Kemmis, tres condiciones son esencialmente necesarias para cualquier proyecto de investigación-acción:

1. El proyecto debe fijar como objetivo una práctica social determinada que debe considerarse susceptible de ser modificada.

2. El proyecto debe caracterizarse por un proceso de indagación constante a través de los ciclos de planificación, acción, observación y reflexión. Cada una de estas fases debe realizarse autorreflexivamente y aplicarse críticamente.

3. El proyecto debe incluir en cada una de estas fases a todas las personas implicadas en una práctica social determinada, y debe incorporar progresivamente a las personas afectadas por esa práctica.

Basándose en la teorfa crítica de Habermas, los mismos autores destacan como la investigación-acción debe rechazar las nociones de racionalidad positivistas y considerar la relación dialéctica entre teoría y práctica; la importancia de las comprensiones de los actores pero, sin embargo, las limitaciones de estas comprensiones como forma suficiente de transformación de la realidad social. Esta visión dialéctica de la realidad social reconoce la yuxtaposición de, por una parte, las restricciones materiales y simbolicas sobre la acción social y, por otra, las restricciones «subjetivas" en la conciencia de los actores, que pueden ser cambiadas a partir de otras formas de conocimiento y comprensión del mundo. A partir de la dialéctica entre acción y reflexión, entre análisis retrospectivo y acción prospectiva, la investigación-acción debe permitir tanto la potenciación de la comprensión de las teorías implícitas en unas determinadas prácticas educativas como potenciar el desarrollo de la capacidad de autocrítica respecto a los factores sociales y contextuales que distorsionan ideológicamente tales prácticas. Es decir, descubrir los principios hegemónicos que limitan unas prácticas sociales determinadas.

Finalmente, la investigación-acción solamente se consolida como ciencia educativa, como ciencia para la educación, si se ponen de relieve aquellos aspec- 
tos del orden social que frustran el cambio racional, y la posibilidad de proporcionar los instrumentos teóricos que permitan al profesorado adquirir consciencia de como pueden ser superados. Ello tiene consecuencias sobre la organización de la acción, es decir, sobre las condiciones a través de las cuales los participantes son impulsados hacia la acción para el cambio. Por ello, Carr y Kemmis subrayan la importancia de un método que ponga de relieve las contradicciones entre las ideas educativas y las prácticas institucionales presentes en la escuela. La investigación-acción, entonces, debe posibilitar la identificación de esas contradicciones por parte del profesorado, como mecanismo clave para el cambio (Carr, 1989; Winter, 1987). Lo que Habermas llama la "organización de la iluminación" son las condiciones bajo las cuales la organización de la acción debe procurar modificar un conjunto de prácticas institucionales distorsionadas.

Esto plantea entonces importantes decisiones relativas al grado de autonomía de los agentes internos a la institución y a la forma que toma la relación entre agentes internos y externos en el proceso de investigación-acción. Las posiciones de la teoría crítica a este respecto indican la necesidad de que en el "proceso de iluminación" intervengan solamente los participantes; pero más allá de una mera acción individual la investigación-acción es acción social $y$, por lo tanto, es intrínsecamente colaborativa, incorporando a los agentes no solamente vinculados a la práctica educativa, sino también a los agentes afectados por esa práctica. Al insistir sobre la necesidad de una «organización del proceso de iluminación» desde dentro, la investigación-acción crítica quiere evitar una vez más la consideración positivista del saber uobjetivable” y científico como único saber legítimo. En el proceso de investigación-acción, la propia legitimidad del conocimiento se genera a partir de la experiencia y a partir de la reflexión crítica sobre la misma. La participación de cualquier persona externa al grupo solamente es legítima si se convierte en parte del grupo, comparte las responsabilidades y participa como uno más en la espiral autocrítica de planificación, acción, observación y experimentación.

\section{Valoración de la investigación-acción crítica e implicaciones para el cambio educativo}

Las reflexiones de autores que parten de la teoría social crítica para construir una ciencia educativa crítica que se basa en la investigación-acción como forma de aproximación a la realidad educativa, escapan sin duda a los planteamientos ateóricos y acríticos sobre la innovación y el cambio educativo. Al distanciarse de los enfoques positivistas, Carr y Kemmis se alinean con la teoría crítica de Hạbermas, en la medida en que desenmascaran el contenido ideológico de las explicaciones "objetivas" de los fenómenos educativos. Ello les permite, asimismo, considerar la importancia de la actividad humana en la producción y reproducción de situaciones educativas. En efecto, el alejamiento de planteamientos positivistas conileva el rechazo de planteamientos sistémicos de la acción social y del cambio institucional. Por lo tanto, al plantear la posibili- 
dad de cambio a través de la investigación-acción, el enfoque crítico está dotando de capacidad de cambio a los propios actores sociales.

Por otro lado, al criticar el enfoque interpretativo sobre ciencia educativa, la investigación-acción crítica subraya la importancia de las prácticas no conscientes del profesorado, así como la existencia de distorsiones ideológicas en las comprensiones e interpretaciones sobre dichas prácticas educativas. Se trata, por lo tanto, de ir más allá de las significaciones que los propios agentes sociales otorgan a sus actos, más lejos del proceso de desvelamiento de la producción y mantenimiento de las relaciones simbólicas en contextos sociales concretos. Desde esta posición crítica, Carr y Kemmis enfatizan la existencia de factores estructurales que condicionan las acciones sociales y, por consiguiente, delimitan las prácticas educativas, factores que están lejos del control directo del profesorado. Una ciencia educativa crítica debe entonces completar las limitaciones del paradigma interpretativo, analizando las causas, orígenes y resultados de las acciones sociales, y superando el nivel de definición de la realidad que realizan los propios actores en cada situación social.

La investigación-acción crítica, en su conjunto, reúne tres conjuntos de factores que permiten superar algunas de las limitaciones que presentan tanto la teoría de las resistencias como las formas pedagógicas dominantes de aproximación al cambio educativo. En primer lugar, las argumentaciones de la investigación-acción crítica responden en gran medida a las limitaciones politicas que antes señalábamos. Consideran acertadamente la naturaleza política de los objetivos educativos y, en consecuencia, la implicación política directa del profesorado en el establecimiento y consecueción de tales objetivos. Solamente a partir de la concepción de la investigación-acción como praxis emancipadora, la intervención educativa es efectivamente transformadora de las situaciones, comprensiones y hechos educativos. Desde este punto de vista, cualquier enfoque que relegue a un segundo plano el carácter político de la práctica educativa no es realmente un planteamiento de cambio $y$, por consiguiente, no escapa a la ideología educativa dominante.

Por otra parte, al situar en el centro de la investigación-acción la uorganización del proceso de iluminación», Carr y Kemmis y otros autores dan respuesta a una de las mayores limitaciones de la teoría de las resistencias educativas: una propuesta teórica y metodologica que justifica y organiza el papel de los profesores como intelectuales en el cambio educativo. La investigación-acción crítica apunta la importancia del cambio "desde dentro" y sitúa en la espiral autocrítica de acción-observación-reflexión-planificación el mecanismo que posibilita la construcción de prácticas efectivamente antihegemónicas. De este modo, se responde a la cuestión clave de la fijación de las condiciones para el cambio. Unas condiciones que tienen que permitir que emerjan las contradicciones existentes entre la práctica institucional y los objetivos educativos. Solamente se produce un cambio si los propios actores, a partir del proceso de autocrítica, realizan un proceso de descubrimiento de los principios ideológicos dominantes subyacentes a una determinada organización de prácticas. 
La tercera cuestión extraordinariamente positiva de la investigación-acción crítica es que constituye un verdadero intento de contrastación en la práctica de lo que entendemos como autonomfa relativa de la cultura. En efecto, al igual que la teoría de las resistencias, la investigación-acción crítica otorga un nivel de autonomía relativa al campo cultural. En el caso de la educación, la autonomía de la cultura significa poder determinar la posibilidad de producción y reproducción de prácticas materiales y simbólicas que pueden no ser siempre reproductoras del orden social. Esta autonomía se desarrolla a nivel teórico a partir de la utilización del concepto gramsciano de hegemonía, como alternativa a la idea de imposición ideológica como forma de dominación. Como señala Williams, la hegemonía es «un modelo que permite la introducción de variaciones y contradicciones, sus posibles alternativas y los procesos de cambion (Williams, 1976). Al entender la hegemonía como un proceso de saturación de la conciencia de los agentes sociales, la teoría de las resistencias - y por extensión, la investigación-acción crítica- provoca una ruptura de la clásica división marxiana entre base y superestructura. En consecuencia, ya no se trata de considerar las repercusiones de las modificaciones en la base sobre la superestructura, sino de considerar las condiciones materiales de existencia como parte integrante de la cultura que tiene su expresión en la conciencia de los agentes sociales. En este sentido, del mismo modo que la hegemonía es una forma de dominación ideológica, Williams acierta al considerar como ésta incorpora la posibilidad de cambio cultural, y a partir de ésta, la posibilidad de cambio sobre las propias condiciones de existencia.

La virtud de la investigación-acción es, pues, poder contrastar en la práctica la dirección, las características y los resultados de un proceso que se mueve únicamente en el campo cultural, y evaluar a partir de este proceso si puede haber una capacidad humana activa y creativa que haga que la producción de la conciencia vaya en "otra dirección" (Willis, 1986). La investigación-acción sitúa a los agentes sociales ante la posibilidad de desarrollar un proceso de producción cultural, es decir, sitúa a un grupo concreto (el profesorado) ante la oportunidad de utilizar un discurso, unos significados y unas prácticas colectivas de forma creativa, que le permiten pensar y actuar de otra forma.

Sin embargo, si retomamos los interrogantes iniciales, existen todavía algunas cuestiones que la investigación-acción crítica deja sin resolver. Cuestiones básicamente asociadas a lo que he denominado limitaciones sociologicas y culturales de la investigación-acción. El dar respuesta a estas cuestiones es un aspecto clave para la construcción de una metodología de cambio educativo que supere las limitaciones descritas anteriormente y que constituya el complemento necesario a la teoría de las resistencias educativas.

En primer lugar, la investigación-acción crítica no nos dice nada acerca del "impulso inicial" que conduce al profesorado hacia una actitud de disposición a la participación y al cambio de prácticas. Si entendemos que la hegemonía actúa saturando la conciencia de los individuos, èes correcto entonces pensar que el hecho de que el profesorado desarrolle y participe en un proceso de inda- 
gación critica puede arrancar y conducirse sin intervención externa? ¿Puede el profesorado introducirse relativamente de forma espontánea en una reflexión crítica que le conducirá a producir nuevas prácticas no reproductoras del orden social?

Además, si el profesorado se ha visto inmerso en una práctica y un discurso dominante que define y explica la problemática educativa en términos de problemas individuales, ¿Puede automáticamente definir los problemas educativos en términos de problemas de grupos sociales y no de problemas individuales? ¿Puede investigar inmediatamente sobre su realidad cotidiana desde una perspectiva sociológica e interpretar sociológicamente los resultados que obtiene?

En segundo lugar, si una de las características de la hegemonía es la extensión de la cultura dominante como única cultura posible, ¿qué es lo que induce a pensar que en el planteamiento del proceso de investigación-acción el profesorado dispondrá de capacidad para identificar los elementos de otras culturas no dominantes, de clase, raza o género, e integratlos en el currículo? Si bien la investigación-acción crítica subraya el carácter emancipador que debe tener la práctica de la investigación-acción, no indica como puede superarse lo que hemos llamado "ceguera cultural" del profesorado.

Lógicamente, todo esto tiene consecuencias sobre la "organización del proceso de iluminación"; es decir, sobre las condiciones que posibilitan dicho cambio. Tiene, por lo tanto, importantes implicaciones sobre el diseño de la metodología de intervención, y sobre el papel que debe efectuar el agente externo en el proceso de investigación-acción y su relación con los agentes internos de la institución escolar. Solamente especificando estas cuestiones se puede dar respuesta a interrogantes como los planteados al comienzo de este artículo.

\section{Bases para una metodología de intervención para el cambio educativo}

Finalmente, y en función de lo expuesto hasta aquí, voy a enumerar brevemente las condiciones que a mi juicio debe teunir la elaboración de una metodología de acción educativa que tenga por objetivo conducir al profesorado hacia un cambio en la organización y desarrollo de sus prácticas de reproducción social y cultural. Los supuestos teóricos básicos de los que parten tales condiciones siguen siendo tanto la teoría de las resistencias educativas como la investigación-acción crítica, en la medida en que parten de la idea fundamental de que el cambio educativo es posible a partir de cambios en la cultura de los agentes sociales y en la medida en que la forma de alcanzar ese cambio debe ser a través de un proceso de reflexion crítica sobre las propias prácticas sociales. Sin embargo, estas condiciones inrentan aportar elementos que superen las limitaciones de estas teorías para ofrecer una respuesta práctica en términos de acción en la escuela para la igualdad entre los grupos sociales y entre las culturas de estos grupos. Las bases para el desartollo de tal método serían las siguientes. 


\section{Cambios en la conciencia}

En línea con los enfogues teóricos señalados, el método debe tener como objetivo fundamental no la modificación directa de prácticas, sino los cambios en la conciencia de los agentes de transmisión cultural. El hecho de que unas determinadas prácticas educativas puedan ser calificadas como resistencia al orden social dominante depende de como éstas son concebidas pedagógica y políticamente (Anyon, 1981; Whitty, 1985). Depende, por lo tanto, de la comprensión de los propios agentes educativos respecto a las actuaciones que llevan a cabo. Esto supone que el proceso de intervención educativa debe retrasarse y supeditarse siempre al alcance de un determinado nivel de conciencia crítica respecto a la problemática analizada.

\section{Intervención del agente externo}

Dado que el principio de hegemonia da lugar a comprensiones, situaciones y hechos educativos distorsionados, el método de acción debe partir de la crítica de las autocomprensiones, cuestión ya señalada por la investigación-acción crítica. Sin embargo, a esta crítica el profesorado no puede llegar de forma espontánea, precisamente porque el principio de hegemonía actúa a través de dos mecanismos fundamentales: la individualización del conflicto y la homogeneidad cultural. La individualización de la problemática escolar oculta la desigualdad entre los grupos y por lo tanto la comprensión sociológica de como una estructura social desigual se proyecta en las relaciones sociales de la escuela. La homogeneidad cultural ajusta las interpretaciones de los actores sociales a un molde dominante $\mathrm{y}$, por consiguiente, incapacita a esos agentes para la percepción de la diversidad cultural.

El cambio en estos dos sistemas interpretativos de la realidad educativa sólo puede alcanzarse, inicialmente, a partir de una acción externa que proporcione los medios para interpretar la realidad educativa como una proyección de relaciones sociales de poder y de formas específicas de control social que tienen su origen en la sociedad y no en los rasgos psicológicos de los individuos. Es decir, como fase previa al propio proceso de investigación para y sobre la acción, el profesorado precisa alguna formación práctica para observar críticamente su realidad educativa cotidiana. Esto conlleva la necesidad de la existencia de un proceso de investigación para la sensibilización sobre un problema político de desigualdad social y cultural, al que el profesorado no accede espontáneamente, sino solamente a través de un proceso de colaboración externa que "conduzca" a los actores hacia un distanciamiento y objetivación en la valoración de las prácticas que tienen lugar en la escuela.

Por otra parte, en una fase inicial, el agente externo proporciona la legitimidad necesaria para el desarrollo del proceso de cambio. El agente externo es visto como un experto en métodos y técnicas de investigación; es en este sentido un agente metodológico que dispone de conocimientos aplicables a la realidad cotidiana del profesorado. Asimismo, el agente externo es un agente 
comprometido con el objetivo político de cambio. La legitimidad del agente externo no se produce solamente por ser un agente metodológico. El agente externo es también y forzosamente un agente comprometido con los objetivos políticos de la investigación-acción. Esto no significa ni mucho menos que el agente externo imponga el cambio. El cambio es llevado a cabo por los agentes internos de la institución, pero para alcanzar la disposición a la realización de prácticas contrahegemónicas el grupo de maestros y maestras debe ser «conducido» externamente.

\section{Del analisis de la proyección de la desigualdad al análisis de la producción de la desigualdad}

Del mismo modo que el principio de hegemonía actúa a través de la individualización-personalización del conflicto, individualiza la culpabilización de las acciones sociales. Esta culpabilización individual es una fuente de resistencia al cambio, ya que los actores tienden a percibir el proceso de cambio como un cuestionamiento de su profesionalización y de la neutralidad de sus prácticas. Esto supone que el método deba organizarse de modo tal que cuando el profesorado realice la valoración de sus propias prácticas esté en situación de comprender los efectos de tales prácticas en términos de culpabilización colectiva, es decir, como reflejo de la proyección de un discurso dominante y no como defectos de su producción personal. La metodología debe ir entonces del análisis de la proyección de la desigualdad social y cultural al análisis de la producción de desigualdad. En la escuela deben analizarse primero las relaciones de poder en el comportamiento del alumnado, la organización escolar del espacio y del tiempo, la programación curricular, etc., (cuestiones que no son completamente una consectrencia directa de las decisiones de profesorado), antes que los sesgos en las decisiones directas de los actores (como las estrategias pedagógicas a seguir, la interacción con el alumnado o los métodos de evaluación).

En definitiva, deben fijarse las condiciones para que cuando el colectivo acceda al análisis directo del proceso de transmisión cultural esté en situación de desculpabilizarse individualmente y culpabilizarse colectivamente de los efectos de la producción de sus prácticas.

\section{Autonomía progresiva de los agentes internos de la institución}

Un indicador lógico de la efectividad de un método de acción para el cambio educativo es el nivel de autonomía que progresivamente van adquiriendo los agentes internos, tanto respecto a la definición e identificación de nuevos problemas como a la producción de nuevas prácticas contrahegemónicas. La intervención del agente externo, en consecuencia, debe ir disminuyendo a medida que el profesorado adquiere mayor conciencia crítica. El rol del agente externo, en este sentido, debe ir modificándose, desde una posición crftica a una más metodológica, de sistematización de las reflexiones y las hipótesis del profesorado. Asimismo, los mismos instrumentos de análisis, muy pautados e 
introducidos por el agente externo en una fase inicial, son construidos por el propio profesorado a medida que se avanza en la adquisición de conciencia crítica.

\section{Hacer emerger las contradicciones}

Lo que entendemos como hegemonía actúa a través de la ocultación de las contradicciones subyacentes a la lógica del proceso de producción de prácticas. La lógica del discurso y de las prácticas dominantes se caracterizan por incorporar las contradicciones del discurso dominante. En el caso de la escuela democrática del capitalismo avanzado existen dos tipos de contradicciones fundamentales en el nivel cultural, aunque sus manifestaciones revisten muy variadas y diversas formas. Estas son contradicciones integradas en el discurso liberal, fundamental para entender la forma que toman las relaciones sociales dentro del sistema educativo (Bowles y Gintis, 1981).

La primera contradiccion es identificable entre libertad individual e igualdad democrática. Los agentes educativos tienden a integrar sin dificultad el principio político de democracia y justicia social con el de desarrollo y libertad individual. La educación del individuo es el eje fundamental del proceso de enseñanza. La enseñanza-aprendizaje es siempre en última instancia un proceso individualizado. La escuela debe garantizar el desarrollo intelectual de los individuos, atender sus deseos y necesidades para formar individuos bien integrados socialmente. Pero en una sociedad democrática la escuela debe rambién garantizar la igualdad de acceso a los recursos disponibles. El derecho a la educación implica que en la escuela deban existir todos los sistemas de compensación necesarios que garanticen una situación de igualdad.

Este proceso, sin embargo, en el límite, es fuertemente contradictorio. En una situacion de recursos escasos, a mayor libertad individual mayor desigualdad potencial. Sin embargo, esta contradicción no es fácilmente identificada por el profesorado si los grupos dominados no luchan por la disponibilidad de los recursos.

La segunda contradicción identificable en el nivel cultural se sitúa entre homogeneización e igualdad cultural. La escuela ha sido y es una institución de normalizacion cultural. El discurso liberal ha construido una forma de entender la integración cultural a partir de la asimilación a la cultura dominante. Los conceptos de "privación cultural" y de educación compensatoria expresan por sí solos una forma de comprender la igualdad educativa que parte de la corrección de las deficiencias culturales de determinados individuos y grupos sociales (Karabel y Hasley, 1977).

Sin embargo estas ideas dominantes durante la década de los sesenta y de los setenta empiezan a ser cuestionadas durante los años ochenta, especialmente en países en los que los niveles de inmigración alcanzan cotas muy altas (caso de Estados Unidos o de Gran Bretaña, por ejemplo). La presión de los movimientos sociales sobre la estructura y contenido de la enseñanza, la magnitud de las migraciones y un volumen creciente de produccion intelectual 
radical, son factores que comienzan a cuestionar la idea de igualdad cultural como asimilación de las culturas de las minorías a una cultura dominante.

La idea de que diferentes grupos sociales llevan consigo culturas propias, distintas y enriquecedoras, gana legitimidad en el discurso educativo dominante. En la misma medida en que la educación intercultural va convirtiéndose en legítima, el reconocimiento de elementos culturales diferentes y stu infrarrepresentatividad en el currículo y en la vida institucional entra progresivamente en contradicción con una estructura y unos contenidos curriculares y una organización escolar homogénea y rígida.

El método, por lo tanto, debe hacer emerger estas dos contradicciones: la primeta debe hacer notar la existencia de desigualdades de acceso a los recursos físicos y pedagógicos de los diferentes grupos (por razón de clase, género o raza). La emergencia de la segunda contradicción debe consistir en que el profesorado identifique elementos culturales diferentes. Recursos personales y grupales distintos a los dados por descontado, distintas necesidades específicas de los grupos representados en la institución, etc. El método, por lo tanto, debe acentuar la percepción cultural del profesorado para conseguir llevar los elementos de las culturas no dominantes a un nivel de visibilidad institucional.

Es obvio que el nivel de legitimidad de la primera contradicción es mayor que el de la segunda. Por lo tanto, parece una hipótesis plausible considerar que la efectividad del cambio pasa por priorizar la identificación de las desigualdades en el nivel de acceso y utilización de los recursos.

Estas cinco condiciones constituyen a mi juicio las bases fundamentales para el diseńo de un método de inducción de un proceso de cambio educativo contrahegemónico. Un método que tenga por objetivo los cambios en la conciencia y en la capacitación crítica para la acción del profesorado. El desarrollo especifico del mismo deberá ajustarse a los objetivos concretos del grupo social cuya situación se pretenda modificar.

\section{Conclusiones}

La sociología de la educación ha tratado poco la cuestión del cambio educativo. Las teorías de la reproducción, o bien lo han marginado de su análisis o bien han situado la fuente de cambio en el nivel estructural, considerando el cambio en el sistema educativo como reflejo de las necesidades del proceso de acumulación capitalista. En un esquema de este tipo, la posibilidad de considerar a los profesores como agentes productores de conocimiento y de prácticas no hegemónicas simplemente no existe. El marco para un análisis del profesorado como agente de cambio educativo hay que buscarlo en la teorfa de las resistencias educativas, cuya formulación sitúa la fuente del cambio en las propias posiciones sociales contradictorias del profesorado y en las contradicciones identificables en el nivel ideolbgico.

Sin embargo, la teoría de las resistencias es limitada al no proporcionar elementos metodológicos que impulsen, a la vez que permitan, explicar la 
dirección y características de los procesos de cambio y las estrategias que pueda seguir el profesorado. Las propuestas que formula están realizadas a un nivel poco más que teórico. No existe ninguna aportación metodológica específica que sitúe al profesorado en el centro del proceso de cambio. La investigación-acción, en su versión más crítica, al construirse sobre una idea de praxis educativa, constituye un complemento metodológico interesante a los desarrollos de la teoría de la posibilidad, aunque no exento de problemas.

Fijadas las condiciones teóricas sobre el cambio educativo y sobre el papel del profesorado en el mismo, el desarrollar un método que induzca una organización y desarrollo de prácticas educativas diferentes es una tarea importante e interesante para la sociología de la educación. Puede serlo desde un punto de vista político, pero independientemente de la implicación política de los agentes de cambio, la inducción de un proceso de cambio institucional debería constituir un aliciente para cualquier sociólogo. El provocar unas determinadas situaciones sociales y analizar las disposiciones y resistencias de los actores sociales proporciona un marco ideal para el estudio de los límites y las posibilidades del cambio institucional, tanto de las ideologías como de las prácticas. El intervenir sobre la realidad y el análisis de esa intervención ha sido una tarea prácticamente desestimada por la sociología 2 .

Ante el agotamiento de ciertos discursos y teorías, especialmente en el ámbito educativo, es un buen momento para atreverse a cierto riesgo metodológico.

\section{Bibliografía}

ANYON, Jean (1981), "Social Class and School Knowledge». En Curriculum Inquiry vol. 1 I (1): 3-42.

- (1983). "Intersections of Gender and Class: Accomodation and Resistance by Working-Class and Affluent Females to Contradictory Sex-Role Ideologies". En WALKER, Stephen; BARTON, Len (eds.). Gender, Class and Education. Lewes: Falmer Press.

APPLE, Michael W. (1982) (ed). Cultural and Economic Reproduction in Education: Essays on Class, Ideology, and the State. Boston: Routledge and Kegan Paul.

- (1986). Teachers and Texts. A Political Economy of Class and Gender Relations in Education. Nueva York: Routledge.

- (1987). Educación y poder. Barcelona: Paidós/MEC.

BAIl, Stephen J. (1988). "Staff Relations During the Teacher's Industrial Action: Context, Conflict and Proletarianisationn. En British Journal of Sociology of Education, vol. 9 (3): 289-306.

Bourdieu, Pierte; Chamboredon, Jean-Claude; PASSERON, Jean-Claude (1976). El oficio de sociologo. Madrid: Siglo XXI.

2. Las investigaciones de Touraine y su equipo de intervención sociológica (1978; 1983) constituyen una excepción en la sociología. 
Bowns, Samuel; GINTIS, Herbert (1976). Schooling in Capitalist America. Nueva York: Basic Books.

- (1981). "Contradiction and Reproduction in Educational Theory". En DalE, Roger y otros (eds.). Education and the State, vol. 1: Schooling and the National Interest. Lewes: Falmer Press.

Carnoy, Martin; LeVin, Henty M. (1985). Schooling and Work in the Democratic State. Stanford, CA: Stanford University Press.

CARR, Wilfred (1989). uAction Research: Ten Years On». En Journal of Curriculum Studies, vol. 21 (1): 85-90.

- (1990). Hacia una ciencia critica de la educación. Barcelona: Laertes.

CARR, Wilfred; KEMMTS, Stephen (1986). Becoming Critical: Education, Knowledge and Action-Research. Lewes: Falmer Press.

Elliotr, John (1990). La investigación-acción en educación. Barcelona: Morata.

GIDDENS, Anthony (1979). La estructura de clases en las sociedades avanzadas. Madrid: Alianza Universidad.

GIMENO SACRISTAN, José (1983). «El profesor como investigador en el aula: un paradigma de formación de profesores». En Educación y Sociedad, núm. 2.

GiRouX, Henry A. (1981). "Hegemony, Resistance, and the Paradox of Educational Reform". En Interchange on Educational Policy, vol. $12(2-3): 3-26$.

- (1983). Theory and Resistance in Education. A Pedagogy for the Opposition. Nueva York: Bergin \& Garvey.

- (1990). Los profesores como intelectuales. Hacia una pedagogia critica del aprendizaje. Barcelona: Paidós-MEC.

- (1992). "La pedagogía de los límites y la política del postmodernismo". En GirouX, Henry A.; FLECHA, Ramón. Igtaldad educativa y diferencia cultural. Barcelona: El Roure.

GRACE, Gerald (1985). "Judging Teachers: the Social and Political Contexts of Teacher Evaluation». En British Journal of Sociology of Education, vol. 6 (1): 3-16.

Guerrero, Antonio (1990). "iPor qué el profesorado no se sindica?». En Educación y Sociedad, núm. 7: 29-52.

HARGREAVES, Andy (1982). "The Rhetoric of School-Centred Innovation" en Journal of Curriculum Studies vol. 14 (3): 251-266.

HARGREAVES, Andy (1988). "Teaching Quality: a sociological analysis". En Journal of Curriculum Studies, vol. 20 (3): 211-231.

Karabel, Jerome; Halsey, A. H. (1977). Power and Ideology in Education. Nueva York: Oxford University Press.

LAWN, Martin; Grace, Gerald (eds.) (1987). Teachers: The Culture and Politics of Work. Lewes: Falmer Press.

MaSJUAN, Josep M. (1974). Els mestres a Catalunya. Barcelona: Novaterra.

OzGA, Jenny; LAWN, Martin (1988). "Schoolwork: Interpreting the Labour Process of Teaching». En British Journal of Sociology of Education, vol. 9 (3): 323-336.

POPKEWITZ, Thomas $\$$. (1988). Paradigma e ideologia en investigacion educativa. Madrid: Mondadori.

STENHOUSE, Lawrence (1981). Investigación y desarrollo del curriculum. Madrid: Morata. TOURAine, Alain (1978). La Voix et le Regard. París: Le Seuil.

TOURAINE, Alain y otros (1983). El Pats contra el Estado: Luchas Occitanas. Valencia: Diputación de Valencia.

VARELA, Julia; OrTEGA, Félix (1984). El aprendiz de maestro. Madrid: MEC. 
WHITTY, Geoff (1985). Sociology and School Knowledge. Curriculum Theory, Research and Politics. Londres: Methuen.

WILliAMS, Raymond (1976). "Base and Superstructure in Marxist Culrural Theory". En DALE, Roger; EsLAND, Geoff; MACDONAID, Madelaine (eds.). Schooling and Capitalism. A Sociological Reader. Londtes: Routledge and Kegan Paul.

WILLIS, Paul (1977). Learning to Labour; How Working Class Kids Get Working Class Jobs. Sussex: Saxon House.

- (1986). "Producción cultural y teorías de la reproducción". En Educación y sociedad, núm. 5: 7-34.

WINTER, Richard (1987). Action-Research and the Nature of the Social Inquiry: Professional Innovation and Education Work. Aldetshot: Avebuty, Gower. 